\title{
Maturational Conversion of Dendritic Early Endosomes and Their Roles in L1-Mediated Axon Growth
}

\author{
Zofia M. Lasiecka, Chan Choo Yap, Joshua Katz, and $₫$ Bettina Winckler \\ Department of Neuroscience, University of Virginia, Charlottesville, Virginia 22908
}

The function of endosomes is intricately linked to cellular function in all cell types, including neurons. Intriguingly, neurons express cell type-specific proteins that localize to endosomes, but little is known about how these neuronal proteins interface with canonical endosomes and ubiquitously expressed endosomal components, such as EEA1 (Early Endosomal Antigen 1). NEEP21 (Neuronal Early Endosomal Protein $21 \mathrm{kDa}$ ) localizes to somatodendritic endosomes, and downregulation of NEEP21 perturbs the correct trafficking of multiple receptors, including glutamate receptors (GluA2) during LTP and amyloidogenic processing of $\beta$ APP. Our own work implicated NEEP21 in correct trafficking of the axonal cell adhesion molecule L1/neuron-glia cell adhesion molecule (NgCAM). NEEP21 dynamically localizes with EEA1-positive early endosomes but is also found in EEA1-negative endosomes. Live imaging reveals that NEEP21positive, EEA1-negative endosomes arise as a consequence of maturational conversion of EEA1/NEEP21 double-positive endosomes. Interfering with EEA1 function causes missorting of L1/NgCAM, axon outgrowth defects on the $\mathrm{L} 1$ substrate, and disturbance of NEEP21 localization. Last, we uncover evidence that functional interference with NEEP21 reduces axon and dendrite growth of primary rat hippocampal neurons on L1 substrate but not on $\mathrm{N}$-cadherin substrate, thus implicating endosomal trafficking through somatodendritic early endosomes in L1-mediated axon growth.

Key words: endosomes; live imaging; NEEP21; NgCAM; Nsg-1; trafficking

\section{Introduction}

Neuronal trafficking depends on both ubiquitously expressed and neuronal-specific endosomal proteins (Steiner et al., 2005; Ha et al., 2008; Yap et al., 2008; Davidson et al., 2009; Hoogenraad et al., 2010; Lasiecka and Winckler, 2011). Since endocytosis and postendocytic endosomal trafficking are implicated in many physiologically important processes in neurons (e.g., axon outgrowth) as well as in disease, we need to understand how the neuron-specific machinery impinges on and interfaces with ubiquitously expressed endosomal regulators. For example, NHE-6 and NHE-9 mutations link endosomal dysfunction to forms of autism and Christianson syndrome (Ouyang et al., 2013). Also, several familial Alzheimer's disease mutations are genetically linked to endosomal trafficking (Wijsman et al., 2011; Pottier et al., 2012).

\footnotetext{
Received May 4, 2014; revised Sept. 5, 2014; accepted Sept. 15, 2014

Author contributions: Z.M.L., C.C.Y., and B.W. designed research; Z.M.L., C.C.Y., and J.K. performed research; Z.M.L., C.C.Y., J.K., and B.W. analyzed data; Z.M.L. and B.W. wrote the paper.

This work was supported by National Institutes of Health Grant R01NS083378 (to B.W.). Z.M.L. and J.K. received a DoubleHoo Award from the University of Virginia. We thank Drs. Silvia Corvera, Ira Mellman, Michael Ehlers, Jim Casanova, David Castle, and Harald Hirling for generously providing crucial reagents. We also thank the Keck Center for Cellular Imaging and the Center Director Dr. Ammasi Periasamy for guidance with the Leica SP5X confocal microscopy system. Last, we thank all members of the Winckler Laboratory for constructive engagement throughout the duration of this work and critical reading of this manuscript.

The authors declare no competing financial interests.

Correspondence should be addressed to Bettina Winckler, Department of Neuroscience, University of Virginia, 409 Lane Road, Charlottesville, VA 22908. E-mail: BWinckler@virginia.edu.

Z. M. Lasiecka's present address: Department of Pathology and Cell Biology, Columbia University Medical Center, 630 West 168th Street, P\&S12-510, New York, NY 10032

DOI:10.1523/JNEUROSCI.1837-14.2014

Copyright $\odot 2014$ the authors $\quad 0270-6474 / 14 / 3414633-11 \$ 15.00 / 0$
}

NEEP21 [Neuronal Early Endosomal Protein $21 \mathrm{kDa}$ (also called neural-specific gene 1 or Nsg-1)] is a member of a gene family of transmembrane proteins (Muthusamy et al., 2009) found specifically in the Golgi/trans-Golgi network (TGN; Sabéran-Djoneidi et al., 1995) and in somatodendritic endosomes (Steiner et al., 2002). Downregulation of NEEP21 caused missorting of endocytosed L1/neuron-glia cell adhesion molecule (NgCAM; Yap et al., 2008). In addition, the downregulation of NEEP21 led to missorting of GluA2 (Steiner et al., 2002, 2005) and defects in hippocampal LTP (Alberi et al., 2005). Intriguingly, downregulation of NEEP2 1 also led to increased amyloidogenic processing of $\beta \mathrm{APP}$ in cultured cells (Norstrom et al., 2010). Given the crucial cargos affected by NEEP21, we set out to uncover how neuronal early endosomes containing the ubiquitously expressed EEA1 (Early Endosomal Antigen 1) and/or NEEP21 were dynamically organized, and whether EEA1 and/or NEEP21 affected L1-mediated axon outgrowth.

\section{Materials and Methods}

Cell culture. Primary neurons were obtained from embryonic day 18 rat hippocampi as described previously (Yap et al., 2008) and cultured for $8-10 \mathrm{~d}$ in vitro (DIV). Fetuses of either sex were used.

Reagents. The following reagents were used: 8D9 anti-NgCAM hybridoma (National Institutes of Health Developmental Studies Hybridoma Bank), which was coupled to Alexa Fluor-488 and Alexa Fluor-568 according to the manufacturer's instructions (Invitrogen); mouse antiEEA1 antibody (BD Transduction Laboratories); mouse anti-TGN38 antibody (from Dr. J. David Castle, University of Virginia, Charlottesville, VA); anti-NEEP21 antibody and NEEP21-GFP plasmid, which were described previously (Steiner et al., 2002); and anti-myc antibody (Santa Cruz Biotechnology). Secondary antibody reagents were pur- 
chased from Invitrogen. Rat myc-L1 was provided by Dr. D. Felsenfeld (Mount Sinai School of Medicine, New York, NY); GFP-EEA1, RFPEEA1, and GFP-DN-EEA1 plasmids were provided by Dr. Silvia Corvera (University of Massachusetts Medical School, Worcester, MA); and CARab5-GFP was provided by Dr. James Casanova (University of Virginia).

Transfection of neuronal cultures. Neuronal cultures at 8-10 DIV were transfected using Lipofectamine 2000 with $1 \mu \mathrm{g}$ of DNA, 2-3 $\mu \mathrm{l}$ of Lipofectamine 2000 for $60 \mathrm{~min}$; washed; and incubated for $18-24 \mathrm{~h}$ or $2 \mathrm{~d}$, depending on the experiment (Lasiecka et al., 2010).

Immunofluorescence. Cells were fixed in $4 \%$ paraformaldehyde $/ 3 \%$ sucrose/PBS in $50 \%$ conditioned medium at room temperature for $20 \mathrm{~min}$. Coverslips were viewed on a Zeiss Axiovert 200 microscope with a $40 \times$ objective. Images were captured with the Orca cooled CCD camera (Hamamatsu) using Openlab software (Improvision) and processed identically in Adobe Photoshop. For confocal imaging, a Leica SP5 X confocal microscope with a $63 \times$ Planapo water objective and a white light laser (WLL) was used.

Endocytosis assay. Neurons expressing NgCAM or L1-myc for $18 \mathrm{~h}$ were incubated with $8 \mathrm{D} 9$ anti-NgCAM antibodies or anti-myc antibodies for $20 \mathrm{~min}$ at $37^{\circ} \mathrm{C}$, and washed several times before imaging (Yap et al., 2008).

Live imaging. Live imaging was performed as in the study by Lasiecka et al. (2010). For dual live imaging of NEEP21 and EEA1 DIV 8/9 neurons were transfected with NEEP21-mCherry/GFP and GFP/RFP-EEA1 and imaged $24 \mathrm{~h}$ later in a live imaging chamber on a heated stage under 5\% $\mathrm{CO}_{2}$. For dual live imaging of endocytosed $\mathrm{NgCAM}$ and EEA1, neurons were transfected with NgCAM and GFP-EEA1 or RFP-EEA1 plasmids. Twenty-four hours after transfection, cells were incubated with Alexa Fluor-488- or Alexa Fluor-568-coupled anti-NgCAM antibody for 30 min, washed, and imaged. Live imaging was performed on a Zeiss Axiovert using $100 \times$ Planapo oil lenses and a Hamamatsu C9100-13 camera as well as a Leica SP5 X confocal microscope using a $63 \times$ Planapo water objective and WLL. Images from the two channels were obtained sequentially. The shortest possible exposure times were used (usually 50-100 $\mathrm{ms}$ ). One minute movies (as indicated by the arrow next to each kymograph) were taken at a rate of $1 \mathrm{frame} / \mathrm{s}, 10 \mathrm{~min}$ movies with a frame rate of 1 frame $/ 10 \mathrm{~s}$, and $\sim 8$ min movies with a frame rate 1 frame $/ 5 \mathrm{~s}$. Fluorescence recovery after photobleaching (FRAP) was performed on a Leica SP5 X confocal using a $63 \times$ Planapo water objective and WLL and a Zeiss Meta confocal microscope using a $63 \times$ Planapo oil objective with a between-lines sequential scanning mode. Only cells expressing low or moderate amounts of proteins were chosen for imaging to minimize overexpression artifacts. Overexpression levels were on average 1.4-fold for GFP-EEA1.

Neurite outgrowth assay. Dissociated neurons were electroporated using Amaxa with either antisense (AS)-NEEP-GFP or GFP-DN-EEA1 or soluble GFP (control) constructs. Cells were plated on coverslips sequentially coated with poly-L-lysine, anti-human Fc antibody, and L1CAM-Fc or N-cadherin-Fc (R\&D Systems). Cells were fixed $2 \mathrm{~d}$ after plating to assay axon and dendrite outgrowth. The length of processes was measured using ImageJ software on two coverslips per condition (in three to five independent experiments). A total of 157-360 neurons were analyzed. Statistical tests were performed using Prism software.

Image analysis and quantification. The axon/dendrite polarity index was determined by measuring the average L1 surface intensity along axons divided by the average L1 intensity along dendrites (for more details, see Wisco et al., 2003). For colocalization analysis of live imaging data, time-lapse sequences were saved as Volocity files, later exported, and further analyzed in Image (NIH), as in the study by Lasiecka et al. (2010).

Analysis of FRAP experiments was performed by applying line intensity scans to the kymograph in ImageJ and exporting intensity values into Excel. Initial intensity was set to $100 \%$, and the percentage of recovery was calculated from the recovery curve after the recovery plateaued. Initial rates of recovery were determined from linear curve fits through the initial rise phase of the recovery graphs.

Colocalization analysis of fixed confocal images was performed in ImageJ (NIH). The percentage of colocalization was calculated as the ratio between the area of overlapping pixels from the single channel area to the total surface area for all analyzed channels. Each channel was thresholded before colocalization analysis to exclude background signal. For each experiment, 8-17 cells were analyzed. Statistical analysis (Student's $t$ test) of both live imaging data and fixed images was performed in GraphPad Prism software.

\section{Results}

EEA1-negative, NEEP21-positive compartments include endosomes

In primary hippocampal neurons, NEEP21 localized to the TGN (marked with TGN38) and in dispersed dendritic compartments (Steiner et al., 2002). The literature reported either low colocalization (Steiner et al., 2002) or almost complete colocalization of EEA1 and NEEP21 (Hoogenraad et al., 2010). In our own hands, NEEP21 was present in 35-45\% of EEA1-positive early endosomes $(\mathrm{E}+\mathrm{N}+)$, and additionally on EEA1-negative dispersed compartments $(\mathrm{E}-\mathrm{N}+)$ as well as on the TGN (data not shown).

To determine whether the NEEP21-positive, EEA1-negative $(\mathrm{E}-/ \mathrm{N}+)$ compartments were endosomes, we asked whether endocytosed cargos were able to accumulate in the $\mathrm{E}-/ \mathrm{N}+$ compartments by triple staining. Since endocytosed L1/NgCAM colocalized partially with both EEA1 and NEEP21 in double labelings (Yap et al., 2008), whereas endocytosed transferrin did not accumulate highly with NEEP21 (data not shown), we performed an endocytosis assay by incubating live neurons expressing myc-L1 with anti-myc antibody. The endocytosed anti-myc antibody was detected after fixation with a secondary antibody (green), and endogenous EEA1 (blue) and NEEP21 (red) were immunostained (Fig. 1A). Examples of the compartment configurations are shown in Figure $1 B$, demonstrating that endocytosed $\mathrm{L} 1-$ myc could enter all three $\mathrm{E}+/ \mathrm{N}-, \mathrm{E}+\mathrm{N}+$, and $\mathrm{E}-/ \mathrm{N}+$ compartments (Fig. 1C). We also observed $\mathrm{E}-/ \mathrm{N}+$ compartments that did not contain endocytosed L1-myc. These compartments might correspond to TGN-derived carriers (Fig. $1 B$, red outline).

\section{NEEP21-containing endosomes are dynamically connected with EEA1-positive early endosomes}

To understand the relationship between the $\mathrm{E}-/ \mathrm{N}+$ endosomes and canonical early (EEA1-positive) endosomes, we used a constitutively active rab5 (CA-rab5 = rab5Q79L), causing excessive fusion into enlarged endosomes (Wegner et al., 2010), which often appeared doughnut shaped (see also Steiner et al., 2002). The expression of rab5-CA-GFP caused striking accumulation of NEEP21 with CA-rab5-GFP, and NEEP21 showed increased overlap with EEA1 (Fig. $1 D, E$ ). In contrast, NEEP21 colocalization with TNG38 was reduced (Fig. $1 F, G$ ). TGN38 distribution itself was not changed in CA-rab5-GFP-expressing neurons (Fig. $1 F)$. Since expression of CA-rab5-GFP led to coalescence of much of the NEEP21 with EEA1, NEEP21-containing endosomes are dynamically connected with EEA1 early endosomes. In addition, the TGN pool of NEEP21 was greatly diminished and likely became trapped in EEA1-positive enlarged endosomes as well. We note that NEEP21 frequently filled the center of the enlarged endosomes, suggesting that it often localized to internal membranes in addition to the limiting membrane.

\section{EEA1 interference leads to missorting of L1/NgCAM to dendrites}

Downregulation of NEEP21 caused increased missorting of L1/ NgCAM to the dendritic surface and to LAMP1 (lysosomeassociated membrane protein 1)-positive endosomes (Yap et al., 2008). We therefore tested whether interfering with EEA1 function caused similar trafficking phenotypes. We were unable to 

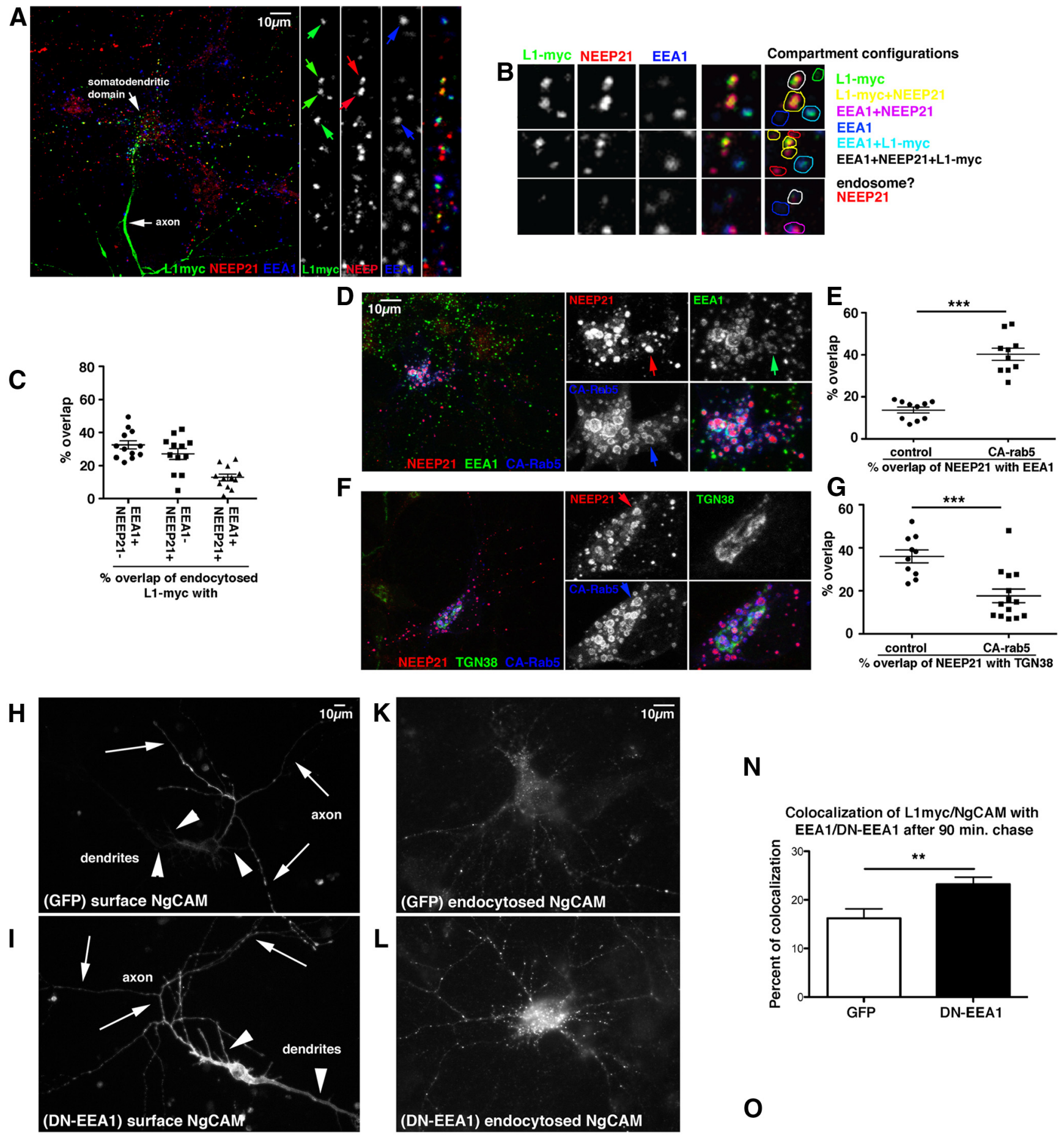

\section{$\mathbf{N}$}

Colocalization of L1myc/NgCAM with EEA1/DN-EEA1 after 90 min. chase
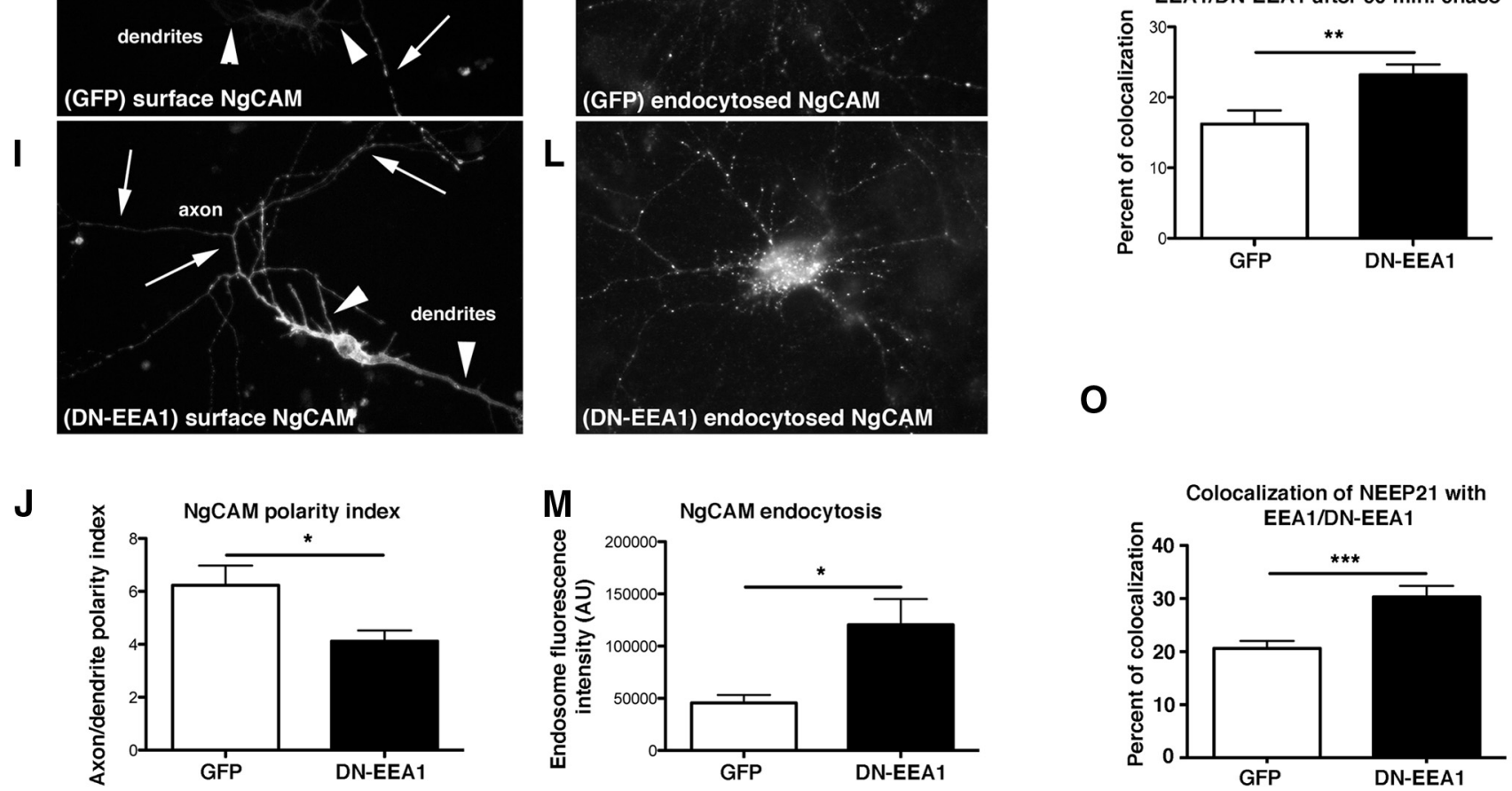

Figure 1. EEA1 interference leads to missorting of L1/NgCAM. A-C, EEA1-negative/NEEP21-positive compartments are endosomes. Neurons were transfected with L1-myc, and endocytosis assays were performed with anti-myc antibodies for 20 min. Endocytosed L1-myc was detected with secondary antibodies (green) and counterstained against NEEP21 (red) and EEA1 (blue). $\boldsymbol{A}$, Overview of one neuron and zoom of a single dendrite. Single channels and triple merges are shown in the side panels. Examples of compartments are shown in detail (Figure legend continues.) 
observe any phenotypes [for either transferrin (Tfn) or L1/NgCAM] when levels of EEA1 were downregulated for $4 \mathrm{~d}$ with a short hairpin plasmid (data not shown), reminiscent of fibroblasts (Lawe et al., 2000, 2002). We note that we previously observed compensation of endosomal trafficking at long time points (Yap et al., 2010). Acute inactivation is thus a preferable approach.

We therefore used a previously characterized EEA1 mutant that acted as a dominant-negative protein for Tfn recycling in fibroblasts (GFP-EEA1-DN; Lawe et al., 2002). GFP-EEA1-DN comprised the C-terminal domain of EEA1, containing the rab5binding and PI3P-binding domains, and was still associated with early endosomes. Neurons were transfected with GFP-EEA1-DN and NgCAM (chick L1) or with GFP-EEA1-DN and L1-myc, depending on subsequent antibody compatibility for counterstaining. The expression of GFP-EEA1-DN for $18-20 \mathrm{~h}$ caused missorting of NgCAM to the dendritic surface (Fig. $1 \mathrm{H}, I$ ), resulting in a decreased axon/dendrite polarity index (Fig. 1J). Interfering with EEAl function thus phenocopied the downregulation of NEEP21. In addition, endocytosed NgCAM accumulated at higher levels in somatodendritic endosomes when GFPEEA1-DN was expressed (Fig. $1 K-M$ ). In particular, endocytosed L1-myc accumulated more highly with EEA1-positive endosomes (Fig. 1N). This phenotype was distinct from NEEP21 downregulation where endocytosed L1/NgCAM accumulated with LAMP1 (Yap et al., 2008). We then wondered whether the localization of NEEP21 was affected by expression of GFP-EEA1DN. EEA1/NEEP21 colocalization was increased (Fig. 1O), but NEEP21 fluorescence intensity was unchanged (data not shown). Inhibiting early endosome progression with GFP-DN-EEA1 therefore led to abnormal accumulation of endocytosed L1/NgCAM and of NEEP21 in somatodendritic early endosomes and to mislocalization of L1/NgCAM to the dendritic surface.

\footnotetext{
$\leftarrow$

(Figure legend continued.) in $\boldsymbol{B}$. The overlap of markers is color coded on the right. $\boldsymbol{C}$, The percentage of overlap of $\mathrm{L} 1$-myc with $\mathrm{E}+\mathrm{N}+, \mathrm{E}-/ \mathrm{N}+$, and $\mathrm{E}+/ \mathrm{N}$ - endosomes was quantified. A scatter plot of individual cell counts is plotted, with means and SEM indicated. Twelve cells were analyzed. D-G, NEEP21 accumulates in enlarged endosomes induced by expression of constitutively active (CA)-rab5. Neurons were transfected with GFP-CA-rab5 (blue) or soluble GFP (control; data not shown) for $24 \mathrm{~h}$, and then fixed and stained against NEEP21 (red) and EEA1 ( $\boldsymbol{D}$, green) or TGN38 ( $\boldsymbol{F}$, green). Single channels and a triple-stained merged image of one soma are shown enlarged in the side panels. Colocalization of NEEP21 with EEA1 $(\boldsymbol{E})$ or TGN38 (G) was quantified and plotted in a scatter plot showing individual cells, as well as mean and SEM. Student's ttest, $p<0.0001$. Ten cells per condition were analyzed. $\boldsymbol{H}-\mathbf{J}$, EEA1 interference leads to missorting of surface $\mathrm{NgCAM}$. Neurons were transfected with $\mathrm{NgCAM}$ and either GFP as control $(\boldsymbol{H})$ or GFP-EEA1-DN $(\boldsymbol{l})$ for $24 \mathrm{~h}$, and then surface $\mathrm{NgCAM}$ was stained in nonpermeabilized cells after fixation. Arrows indicate axons, and arrowheads indicate dendrites. The average $\mathrm{NgCAM}$ surface staining intensity was determined for GFP-transfected cells ( $n=24$ cells) and GFP-DN-EEA1 ( $n=38$ cells) along the axon and dendrites, and the axon/dendrite polarity index was calculated (J). Bar indicates the SEM. ${ }^{*} p<0.01$, Student's $t$ test. One representative experiment is shown (from a total of three independent experiments). $\boldsymbol{K}-\boldsymbol{N}$, Endocytosed $\mathbf{~ N g}$ CAM accumulates in somatodendritic endosomes when EEA-DN is coexpressed. Neurons were transfected with NgCAM and either GFP as control $(\boldsymbol{K})$ or GFP-EEA1-DN $(\boldsymbol{L})$ for $24 \mathrm{~h}$ and anti$\mathrm{NgCAM}$ antibody was fed to live cells for $20 \mathrm{~min}$. Endocytosed $\mathrm{NgCAM}$ was detected with a secondary antibody after acid stripping of surface-bound antibody. The extent of endocytosed $\mathrm{NgCAM}$ in somatic endosomes was quantified (M). $N=47$ cells (GFP), 51 cells (EEA1-DN). Bar indicates the SEM. ${ }^{*} p<0.01$, Student's $t$ test. One representative experiment is shown (from a total of three independent experiments). $\boldsymbol{N}$, Endocytosed L1-myc accumulated significantly in EEA1-positive endosomes after 90 min of endocytosed L1-myc antibody chase. $N=21$ cells (GFP), and $N=34$ cells (DN-EEA1). Bar indicates the SEM. ${ }^{* *} p<0.001$ Student's $t$ test. One representative experiment is shown (from a total of three independent experiments). 0 , 0verexpression of GFP-EEA1-DN in neurons caused increased accumulation of NEEP21 in EEA1 endosomes. $N=17$ cells (GFP), and $N=12$ cells (DN-EEA1). Bar indicates the SEM. ${ }^{* *} p<$ 0.0001 , Student's $t$ test.
}

\section{Interference with EEA1 or NEEP21 results in decreased axon and dendrite growth}

We next tested whether aberrant endosomal trafficking of L1/ NgCAM affected the ability of cultured neurons to extend processes on the L1-Fc substrate. The lengths of dendrites and of the major axonal process were measured after 2 DIV in control cells expressing GFP (Fig. 2A,D) or cells expressing either antisenseNEEP21-GFP (Fig. 2B; Yap et al., 2008) or GFP-EEA1-DN (Fig. $2 E$ ). For both AS-NEEP21-GFP (Fig. 2C) and GFP-EEA1-DN (Fig. $2 F$ ), axon lengths as well as total dendrite lengths were reduced. The mean dendrite length was not changed, and the decrease in total dendrite length was due to a small decrease in the average number of dendrites. Since both EEA1 and NEEP21 are present only in dendrites, somatodendritic endosomes are functionally important for correct trafficking of axon outgrowth receptors and growth of neuronal processes.

We then asked whether NEEP21 also affected axon and dendrite growth on other substrates. We coated coverslips with $\mathrm{N}$-cadherin-Fc and plated neurons transfected with GFP or ASNEEP21-GFP for 2 d. GFP-expressing control neurons and AS-NEEP21-GFP expressing neurons showed no statistically significant changes in axon length on $\mathrm{N}$-cadherin-Fc (average axon length normalized to GFP controls $=90 \%$; SEM $=7 \% ; n=5$ independent experiments; $p=0.22$ ). Dendrite number was also not significantly affected (average dendrite number normalized to GFP controls $=97 \%$; SEM $=4 \% ; n=5$ independent experiments; $p=0.55$ ). NEEP21 thus affected process growth preferentially on L1-Fc substrate.

\section{Live imaging of NEEP21-positive endosomes reveals a large, motile pool that is EEA1 negative}

To unravel the dynamic relationships between NEEP21- and EEA1-positive early compartments, we performed dual live imaging (Fig. 3). GFP-EEA1 and NEEP21-mCherry were cotransfected into 8/9 DIV neurons and imaged in a live chamber $24 \mathrm{~h}$ later (frame rates: 1 frame/s, 1 frame $/ 5 \mathrm{~s}$, or 1 frame $/ 10 \mathrm{~s}$ ). The tagged overexpressed proteins recapitulated the partial colocalization of endogenous NEEP21 with EEA1 (Fig. $3 A, B$ ). The motile behavior of the EEA1- and NEEP21-positive compartments was strikingly different (Fig. 3A; Movie 1). EEA1 rarely moved whether or not NEEP21 was also present in the same compartment (Fig. 3B). NEEP21, on the other hand, showed frequent and often long-distance movements, both anterogradely and retrogradely. EEA1 movements were not only rare, they were also mostly short distance $(<5 \mu \mathrm{m})$ and slow. NEEP21 movements, on the other hand, were often over long distances (tens of micrometers) and could be as fast as $1.7 \mu \mathrm{m} / \mathrm{s}$, similar to the fast moving endosomes we previously observed (Lasiecka et al., 2010). EEA1-positive endosomes thus were mostly stationary, and it was a subset of these stationary EEA1-positive compartments that contained NEEP21. NEEP21-positive compartments were frequently motile, and these motile NEEP21-positive compartments rarely contained EEA1.

\section{Dynamic relationship between EEA1 and NEEP21 compartments: putative fusion events}

Next, we wondered whether the classes of compartments we observed, $\mathrm{E}+/ \mathrm{N}-, \mathrm{E}+\mathrm{N}+$, and $\mathrm{E}-/ \mathrm{N}+$, might be serially related to each other. Several possibilities (not mutually exclusive) came to mind: $\mathrm{E}+\mathrm{N}+$ compartments might be generated from $\mathrm{E}+/ \mathrm{N}-$ compartments by the fusion of $\mathrm{N}+$ vesicles, or $\mathrm{E}+\mathrm{N}+$ compartments might be generated from $\mathrm{E}-/ \mathrm{N}+$ compartments by recruiting EEA1 from a cytosolic pool. In addition, single positive 
A

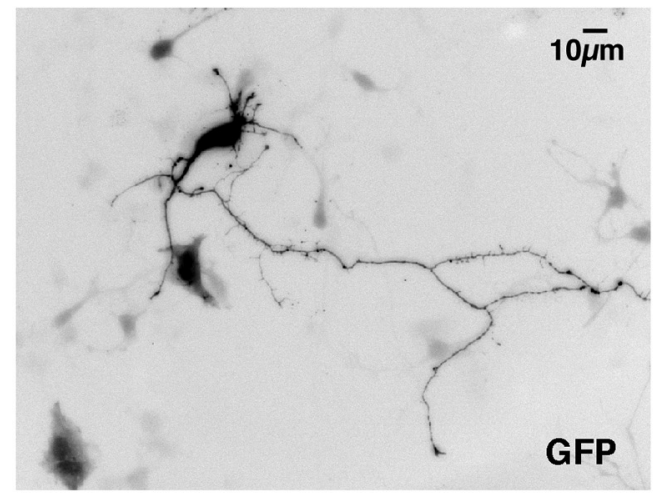

B

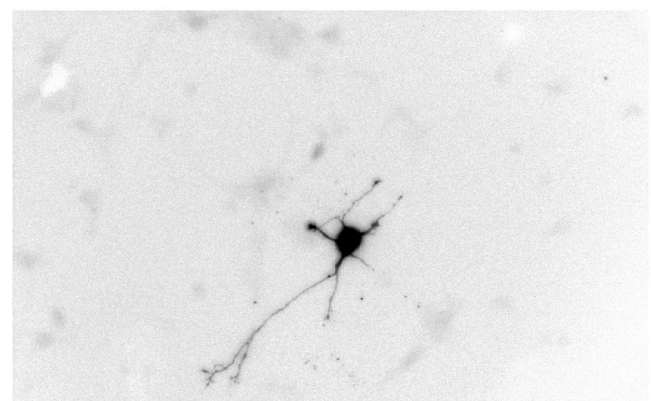

AS-NEEP21

C

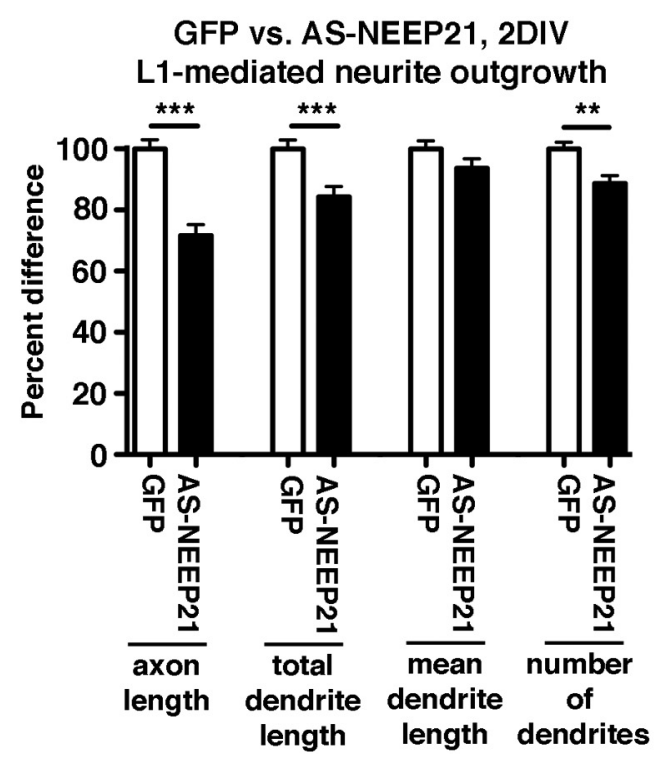

D

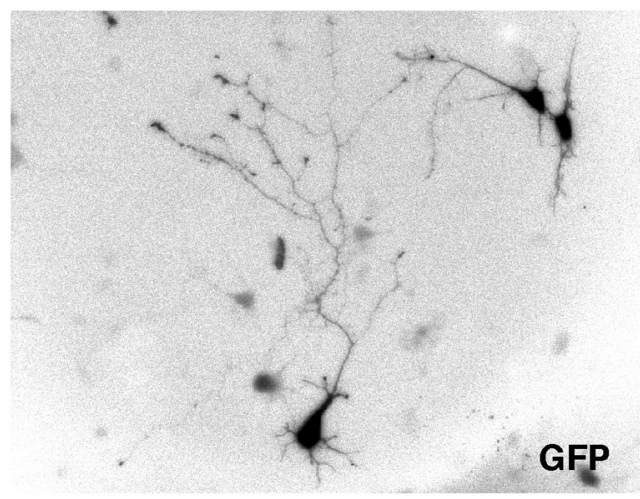

E

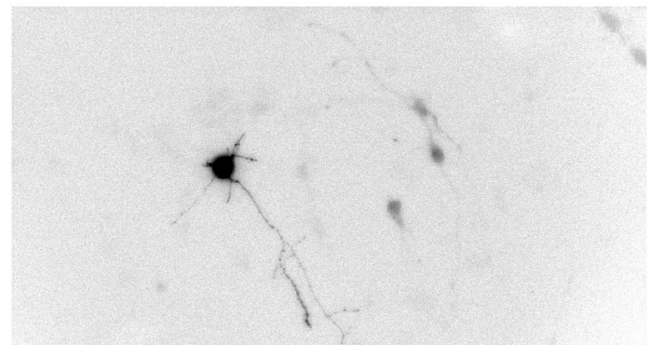

DN-EEA1

$\mathbf{F}$

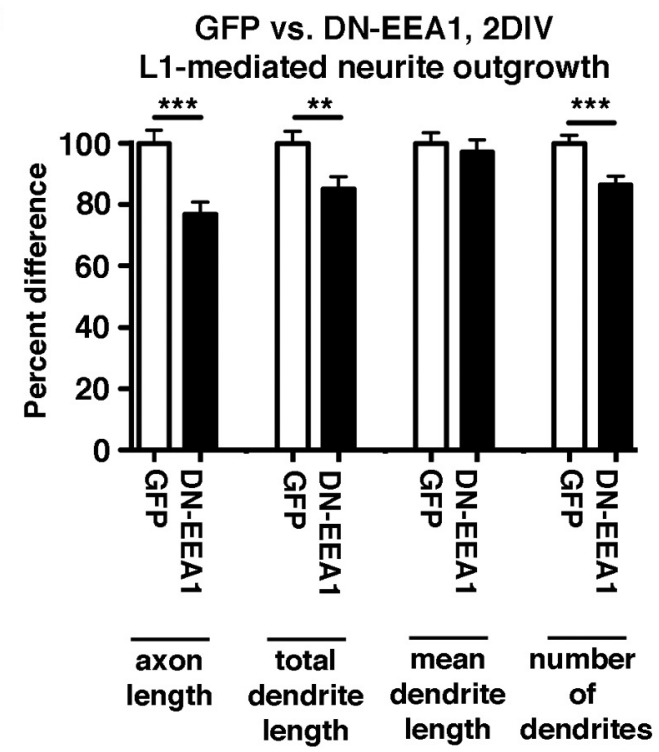

Figure 2. Interference with EEA1 or NEEP21 results in decreased axon and dendrite growth. $A-C$, Downregulation of NEEP21 results in decreased axon and dendrite growth on L1-Fc. Freshly dissociated hippocampal neurons were electroporated with GFP $(\boldsymbol{A})$ or GFP-AS-NEEP21 (B) and plated on L1-Fc-coated coverslips. The length of the longest process ("axon length"), the combined length of all minor processes ("total dendrite length"), the average dendrite length ("mean dendrite length"), and the number of primary dendrites were measured (after $2 \mathrm{~d}$ in culture) and were normalized to control values for easier comparison (C). A total of 190-360 cells were quantified (five independent experiments). Bar indicates the SEM. ${ }^{* *} p<0.001 ;{ }^{* *} p<0.0001$. $\boldsymbol{D}-\boldsymbol{F}$, Expression of GFP-EEA1-DN results in decreased axon and dendrite growth on L1-Fc. Freshly dissociated cortical neurons were electroporated with GFP (D) or GFP-EEA1-DN (E), and were plated on L1-Fc-coated coverslips. The length of the longest process ("axon length"), the combined length of all minor processes ("total dendrite length"), the average dendrite length ("mean dendrite length"), and the number of primary dendrites were measured $(\boldsymbol{F})$. A total of $157-187$ cells were quantified (three independent experiments). Bar indicates the $S E M .{ }^{* *} p<0.001,{ }^{* *} p<0.0001$.

compartments might be downstream of $\mathrm{E}+\mathrm{N}+\mathrm{E}+\mathrm{N}+$ might become $\mathrm{E}+/ \mathrm{N}-$ by the budding of $\mathrm{N}+$ vesicles or $\mathrm{E}+\mathrm{N}+$ might become $\mathrm{E}-/ \mathrm{N}+$ by dissociation of EEA1. Events consistent with such behaviors can in principle be observed by live imaging. We thus analyzed two types of dynamic events quantitatively from dual live imaging data: (1) $\mathrm{E}+/ \mathrm{N}-$ compartment converting to $\mathrm{E}+\mathrm{N}+$ by putative fusion of $\mathrm{N}+$ vesicles (Fig. 3); and (2) EEA1 conversion events in which either $\mathrm{E}-/ \mathrm{N}+$ compartments be- came $\mathrm{E}+\mathrm{N}+$ by recruiting EEA1 or $\mathrm{E}+\mathrm{N}+$ compartments became $\mathrm{E}-/ \mathrm{N}+$ by loss of EEAl (Fig. 4).

Putative fusions were defined as events where a motile vesicle encountered a stationary vesicle and failed to emerge from it on the other side for the rest of the kymograph. Since it is extremely difficult to prove a true fusion event, we refer to these events as "putative fusions." The alternative interpretation of such events is that the motile vesicle stopped at the exact same position of a 
A

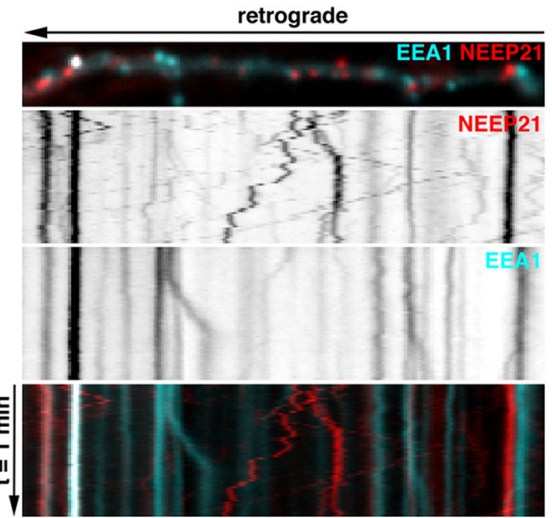

B

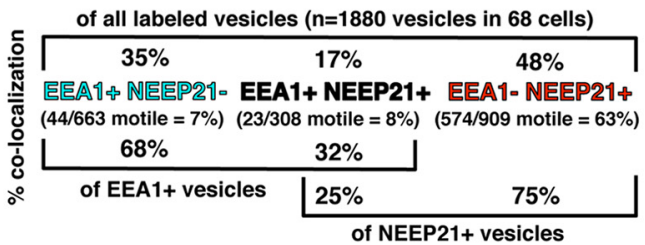

C putative fusion events (20 fusions/559 motile vesicles)

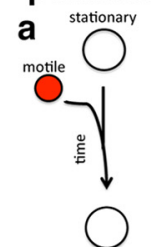

12 events

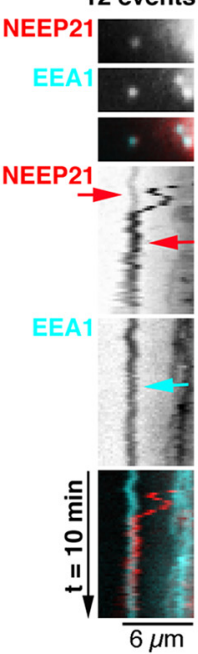

b
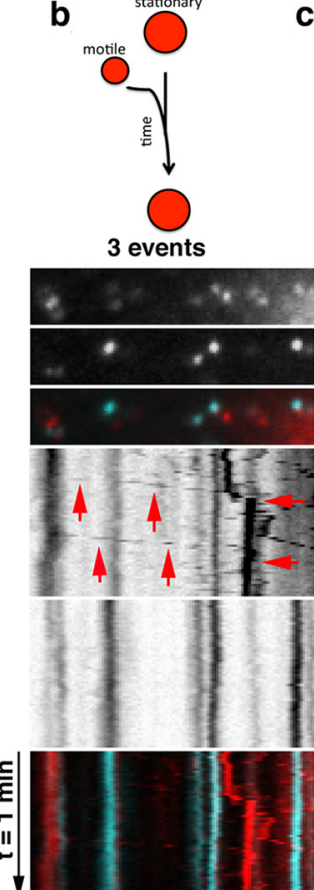

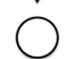

5 events

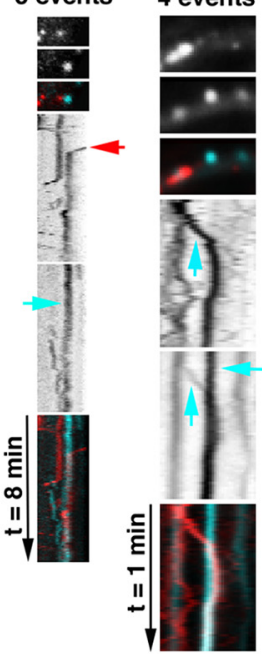

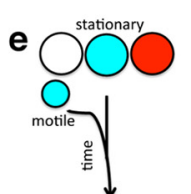

$\bigcirc$

4 events

D FRAP (EEA1 bleach: 45/53 recover; NEEP21 bleach: 7/52 recover)
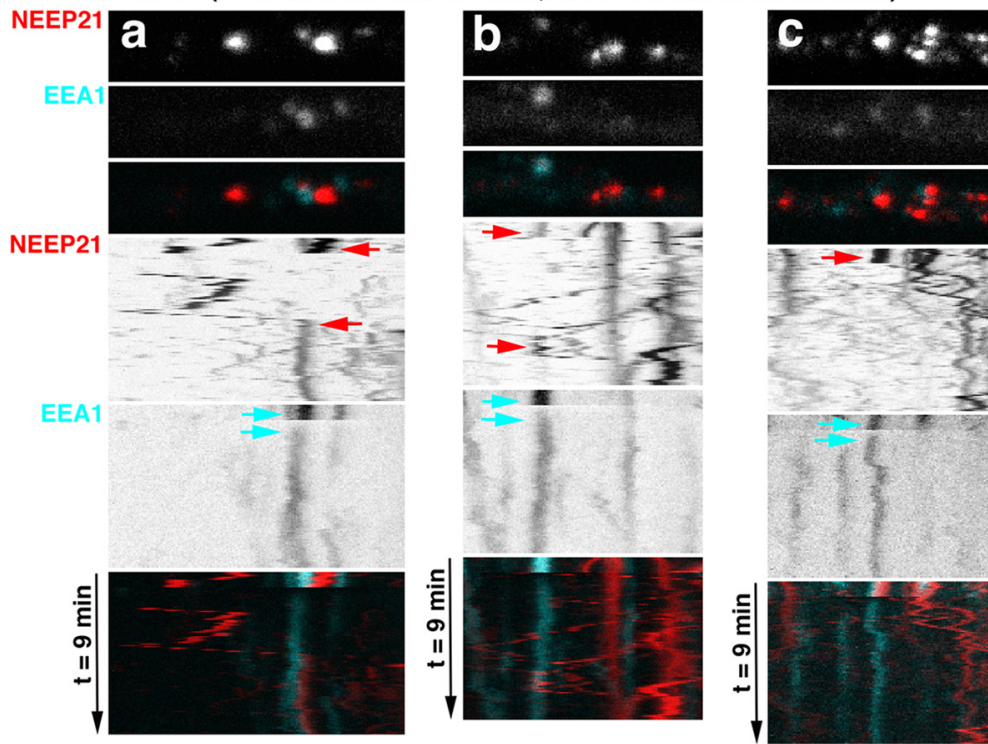
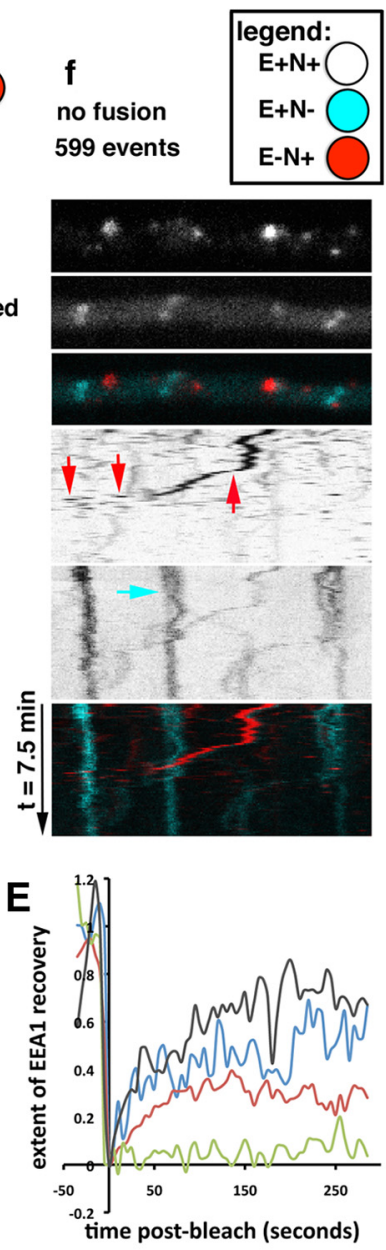

$F$

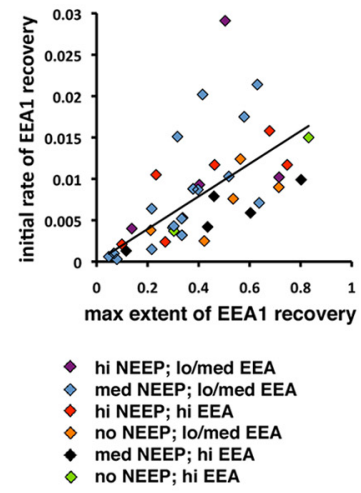


$\rightarrow$

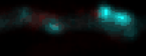

$\left(\frac{10}{2}\right.$

Movie 1. The movie corresponds to Figure $3 A$. NEEP21-mcherry and GFP-EEA1 were imaged in a DIV $9 / 10$ neuron. The acquisition frame rate is 1 frame/s. Playback is 7 frames/s.

stationary endosome, but did not fuse with it. Analysis of hundreds of vesicles revealed that the vast majority of motile vesicles passed stationary endosomes without stopping (Fig. 3C, f). For $\mathrm{E}-/ \mathrm{N}+$, motile vesicles (which comprised $88 \%$ of motile vesicles), 97\% (539 of 559 motile vesicles) passed stationary endosomes unimpeded. Mere steric hindrance did therefore not appear to be a frequent reason for stopping at the precise position of a stationary vesicle. Of the 20 motile $\mathrm{E}-/ \mathrm{N}+$ vesicles whose behavior fit the criteria for putative fusions, $12(60 \%)$ fused with a stationary $\mathrm{E}+\mathrm{N}+$ compartment (Fig. 3C, a), 3 (15\%) fused with a stationary $\mathrm{E}-/ \mathrm{N}+$ compartment (Fig. 3C, $b$ ), and $5(25 \%)$ fused with a stationary $\mathrm{E}+/ \mathrm{N}-$ compartment (Fig. $3 C, c$ ). $\mathrm{E}+\mathrm{N}+$ compartments were rarely motile (30 of 623 compartments; $5 \%$ ), but occasionally behaviors consistent with putative fusions were observed [ 4 of $30 \mathrm{E}+\mathrm{N}+$ motile vesicles (13\%) underwent putative fusion events; Fig. $3 C, d]$. $\mathrm{E}+/ \mathrm{N}-$ compartments were also rarely motile [ 38 of 629 of all motile compartments $(6 \%)]$ and were never observed to engage in putative fusions (Fig. $3 C, e$ ). Examples of observed putative fusions are shown in Figure $3 C(a-f)$.

Putative fusion events were sometimes difficult to observe because of the high density of stationary endosomes. We thus photobleached stationary endosomes ( 86 vesicles in 35 cells) and imaged for $8 \mathrm{~min}$ (frame rate: 1 frame $/ 5 \mathrm{~s}$ ) to observe whether fluorescence of the photobleached endosome would recover (FRAP). Since NEEP21 is a transmembrane protein, recovery required the delivery of NEEP21 in a vesicle. When 52 NEEP21-

\section{$\leftarrow$}

Figure 3. Live imaging of EEA1- and NEEP21-positive endosomes in dendrites reveals putative fusion events and differential stability of EEA1 on endosomes. $A$, Live imaging was performed in cultured neurons transfected for $24 \mathrm{~h}$ with GFP-EEA1 (turquoise) and NEEP21$\mathrm{mCherry}$ (red). Kymographs were prepared from the movies, and one dendrite is shown as an example. A still frame from the movie is shown on top with the direction of retrograde movement indicated by an arrow. Single channels are shown as inverted black-and-white images on top of the merged kymographs (see Movie 1). Quantification of colocalization of all labeled endosomes, and separately for motile endosomes, is shown in $\boldsymbol{B}$. The brackets at the bottom of the graph show the quantification of colocalization, whether the point of view of one marker is taken rather than that of all stained endosomes. C, Kymographs of live imaging in GFP-EEA1/ NEEP21-mcherry-expressing neurons were analyzed for putative fusion events. Putative fusion events were rare (20 of 559 motile vesicles). The kinds of putative fusion events are indicated by the diagram: red $=\mathrm{E}-/ \mathrm{N}+$ vesicle; turquoise $=\mathrm{E}+/ \mathrm{N}-$ vesicle; white $=\mathrm{E}+\mathrm{N}+$ vesicle. Kymographs illustrating an example of each case are shown below the diagrams: 12 events of $\mathrm{E}-\mathrm{N}+$ vesicle undergoing putative fusion with stationary $\mathrm{E}+\mathrm{N}+(\boldsymbol{a}) ;(\boldsymbol{b}) 3$ events of $\mathrm{E}-/ \mathrm{N}+$ vesicle undergoing putative fusion with stationary $\mathrm{E}-/ \mathrm{N}+(\boldsymbol{b}) ; 5$ events of $\mathrm{E}-\mathrm{N}+$ vesicle undergoing putative fusion with stationary $\mathrm{E}+\mathrm{N}-(\mathbf{c}) ; 4$ events of $\mathrm{E}+\mathrm{N}+$ vesicle undergoing putative fusion with stationary $\mathrm{E}+/ \mathrm{N}-(\boldsymbol{d}) ; n$ no events of motile $\mathrm{E}+/ \mathrm{N}$ - vesicles fusing $(\boldsymbol{e})$; and example of motile NEEP21-vesicle moving past stationary endosomes unimpeded $(\boldsymbol{f})$. D, FRAP experiments were performed on neurons expressing GFP-EEA1 and NEEP21mcherry. Arrows indicate the bleach event and the recovery event. $\boldsymbol{a}-\boldsymbol{c}$, Examples of different post-FRAP behaviors. Movie 2 corresponds to the dendrite shown in $\boldsymbol{a}$. $\boldsymbol{E}$, GFP-EEA1 recovery after FRAP was extensive (45 of 53 recovered). GFP-EEA1 recovery was quantified after FRAP, and four examples of GFP-EEA1 recovery are plotted. The black line exemplifies one of the highest observed recoveries. The green line exemplifies one of the low/no recoveries. The blue and red lines are intermediate in recovery. $\boldsymbol{F}$, The maximal recovery extent correlates positively with the initial rate of recovery. No correlation was found with the recovery rate or extent of recovery based on how much GFP-EEA1 or NEEP21-mCherry were initially present on the endosome before the FRAP. Color coding is indicated. positive endosomes were photobleached, recovery was observed seven times. Motile NEEP21 vesicles were observed to traverse the position of the photobleached vesicle in 45 cases without stopping (Fig. $3 D, c)$. In the seven recoveries, a NEEP21-positive vesicle arrived and then stopped at the position of the bleached compartment (Fig. 3D, $a$; Movie 2). Six recoveries occurred with $\mathrm{E}-/ \mathrm{N}+$ compartments (of 26 photobleached $\mathrm{E}-/ \mathrm{N}+$ compartments), and the seventh recovery occurred with an $\mathrm{E}+\mathrm{N}+$ compartment (of 26 photobleached $\mathrm{E}+\mathrm{N}+$ compartments). In three cases, motile NEEP21-positive vesicles approached and temporarily halted at the position of the bleached compartment, but ultimately the vesicle moved away (Fig. $3 D, b$ ). FRAP analysis thus revealed putative fusion events of NEEP21-containing vesicles with stationary $\mathrm{E}-/ \mathrm{N}+$ and $\mathrm{E}+\mathrm{N}+$ compartments, but the occurrence was low.

\section{EEA1 recovery after photobleaching reveals dynamic recruitment onto endosomes}

FRAP analysis was also performed in 53 EEA1-positive endosomes, and the recovery of EEA1 was observed in 45 of them. In Figure $3 D, c$, as an example, four EEA1-positive endosomes were photobleached, and three of them recovered some EEA1 fluorescence. Forty of 53 FRAP events were completely bleached to background, and the dynamics of EEA1 recruitment onto early endosomes was analyzed quantitatively. Intensity line scans revealed a range of recovery rates and extents (Fig. 3E). Some endosomes rapidly recovered to a high level of EEA1 fluorescence. The highest initial rate of recovery was 3\% fluorescence/s, and the highest extent of recovery was $81 \%$. Others only recovered a small percentage of their initial EEA1 fluorescence (10-30\%). When the maximum recovery extent was plotted against the initial rate of recovery for all 40 FRAP events, a positive correlation was found that was statistically significantly different from zero $(p<$ $0.0001)$ : the vesicles that recovered the most fluorescence did so with a faster initial rate than the vesicles that recovered only a small percentage of their initial fluorescence (Fig. 3E). Therefore, we observed different populations of EEA1-positive endosomes. Some populations were very stable, and very little EEA1 exchanged slowly onto the endosomes from the soluble pool. Other populations rapidly exchanged EEA1 from the soluble pool. We then determined whether the presence of NEEP21 correlated with either the more stable or the more dynamic subpopulations of EEA1-positive endosomes. The initial EEA1 and NEEP21 fluorescence intensity before photobleaching was determined and color coded onto Figure 3F. No differences in dynamic behavior were apparent between the endosomes that contained initially high or low NEEP21, or high or low EEA1.

\section{Dynamic relationship between EEA1 and NEEP21 compartments: putative maturation events}

While analyzing kymographs, we noticed that the EEA1 signal sometimes changed intensity and became either dimmer or brighter. These behaviors suggested that EEA1 might either recruit onto or dissociate from endosomes, allowing us to monitor maturation of EEA1- and NEEP21-positive compartments. We thus analyzed all NEEP21/EEA1 kymographs for such spontaneous conversion events in which a NEEP21-positive compartment $(\mathrm{E}-/ \mathrm{N}+)$ either converted to an EEA1-positive compartment $(\mathrm{E}+\mathrm{N}+)$, or a double-positive compartment $(\mathrm{E}+\mathrm{N}+)$ lost EEA1 to convert to $\mathrm{E}-/ \mathrm{N}+$. Only events in which NEEP21 fluorescence remained largely unchanged were included. In a few cases, NEEP21 fluorescence dimmed and the trace widened. These 


\section{A EEA1 conversion events}

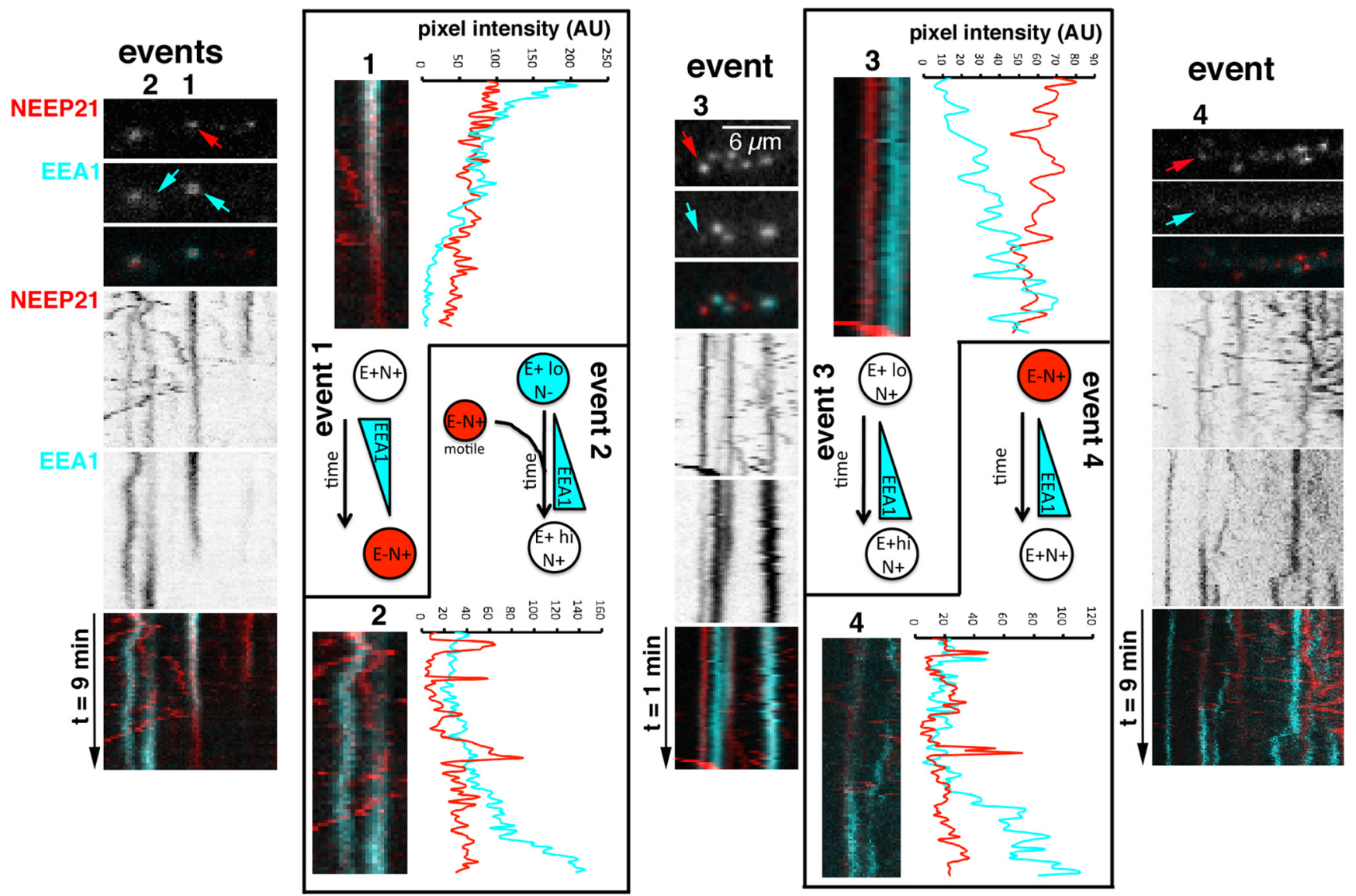

B
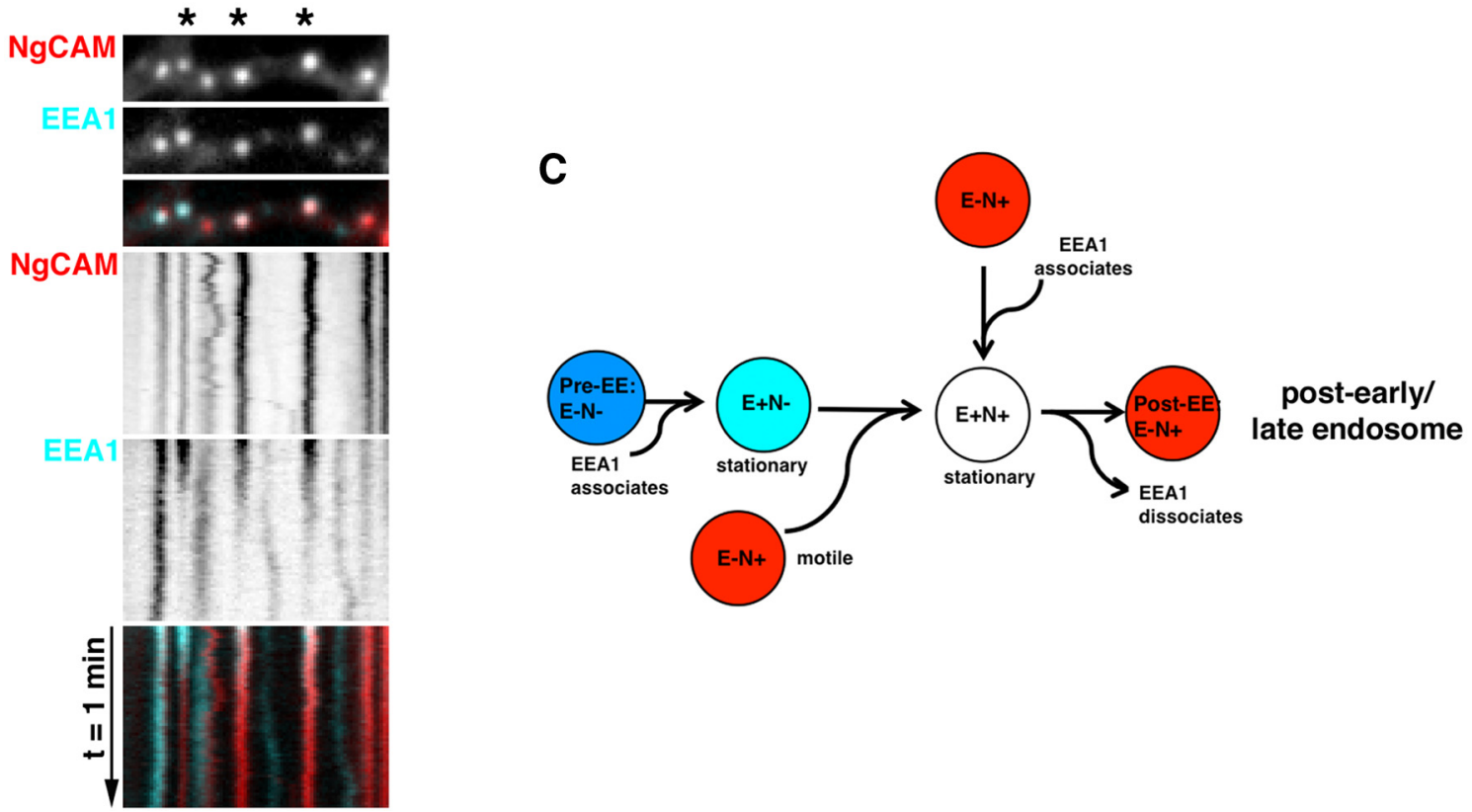

Figure 4. EEA1 dynamically associates and dissociates from NEEP21 endosomes. A, Kymographs of GFP-EEA1/NEEP21-mCherry-expressing neurons show that events were EEA1 associates or dissociates from endosomes ("conversion events"). Examples of the kinds of conversion events are illustrated and are numbered 1-4 above the observed endosome (marked with arrows). For each example, a zoomed-in closeup of the converting endosome is shown together with a diagram illustrating the observed conversion and an intensity scan of the conversion event. $\boldsymbol{B}$, EEA1 conversion events can be observed on endosomes containing endocytosed NgCAM. Stars above the kymograph indicate the EEA1-containing endosomes, which show conversion to EEA1-negative, NgCAM+ endosomes (Movie 3). C, Endosomal maturation of EEA1-positive endosomes in dendrites. We propose a model in which $\mathrm{E}+\mathrm{N}+$ endosomes can arise by the following two pathways: EEA1 associates with $\mathrm{E}-/ \mathrm{N}+$ endosomes; and/or motile $\mathrm{E}-\mathrm{N}+$ vesicles fuse with EEA1 + endosomes. $\mathrm{E}+\mathrm{N}+$ endosomes can convert to $\mathrm{E}-/ \mathrm{N}+$ endosomes by dissociation of EEA1 to result in post-EE $\mathrm{E}-/ \mathrm{N}+$ endosomes. 


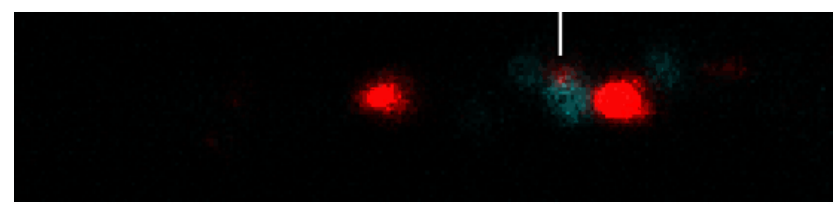

Movie 2. The movie corresponds to Figure $3 \boldsymbol{D}, \boldsymbol{a}$. NEEP21-mcherry and GFP-EEA1 were imaged in a DIV 9/10 neuron. The acquisition frame rate is $1 \mathrm{frame} / 5 \mathrm{~s}$. Playback is 7 frames $/ \mathrm{s}$. FRAP was performed (where the movie suddenly becomes dark). The white line marks the position of endosomes that recover GFP-EEA1 and NEEP21-mcherry.

events likely corresponded to the slight loss of focus of the endosomes and were therefore not included in the analysis.

Of $1097 \mathrm{E}+\mathrm{N}+$ stationary endosomes in 128 kymographs, 97 changed EEA1 intensity over the time course of $10 \mathrm{~min}$. Sixty-two percent of the observed EEA1 intensity changes corresponded to diminution in staining. For most of these, the change in intensity was partial. Twelve of 97 events changed EEA 1 intensity by $>80 \%$ (i.e., a mostly complete conversion event was captured in the 10 min kymograph). This low rate of complete EEA1 conversions indicated that the dendritic EEA1-positive compartments were mostly stable and long lived. Intensity line scans were performed on the 12 complete conversions. Eight of them showed loss of EEA1 (conversion from $\mathrm{E}+\mathrm{N}+$ to $\mathrm{E}-/ \mathrm{N}+$; Fig. $4 A$, event type 1 , arrow). $\mathrm{E}-/ \mathrm{N}+$ compartments could therefore be generated from $\mathrm{E}+\mathrm{N}+$ compartments by a maturation event involving the apparent dissociation of EEA1. The other four conversion events showed increases in EEA1 intensity. In one case, we observed an $\mathrm{E}-/ \mathrm{N}+$ compartment converting to $\mathrm{E}+\mathrm{N}+$ by apparent recruitment of EEA1 (Fig. $4 A$, event 3, arrow). E-/N+ compartments could thus mature to $\mathrm{E}+\mathrm{N}+$ double-positive endosomes, but this was observed much less frequently than the loss of EEA1 from an $\mathrm{E}+\mathrm{N}+$ double-positive endosome. In another case (Fig. $4 A$, event 4 , arrow), a NEEP21 vesicle, which had dim EEA1 staining to start with, abruptly increased EEA1 intensity greatly. In two cases, EEA1 gradually recruited onto a location where there was no NEEP21 initially. Active NEEP21 vesicle transport could be observed at the location of the endosome. These crossing NEEP21 vesicles gave rise to peaks on the intensity scans (Fig. $4 A$, event type 2 ). At the end of the time line, the vesicle had converted to $\mathrm{E}+\mathrm{N}+$. The increase in NEEP21 might have resulted from vesicles fusing and delivering some NEEP21 to the maturing EEA1+ vesicle. We did not observe subsequent changes in motility of postconversion NEEP21-positive endosomes. All but one remained stationary for the rest of the imaging period.

Since we observed clear examples of maturation of $\mathrm{E}+\mathrm{N}+$ endosomes to $\mathrm{E}-/ \mathrm{N}+$ endosomes, we wondered whether endocytosed cargo, such as L1/NgCAM would remain associated with NEEP21 after EEA1 dissociation. We thus performed live imaging of endocytosed L1/NgCAM (labeled with Alexa Fluor-568anti-NgCAM antibody) and GFP-EEA1 (Fig. 4B; Movie 3). The colocalization of endocytosed L1/NgCAM after 30 min of antibody uptake with stationary GFP-EEA1 endosomes was 51\%. EEA1 conversion events where EEA1 was lost from a stationary endosome were frequently observed (Fig. $4 B$, asterisks). Endocytosed $\mathrm{L} 1 / \mathrm{NgCAM}$ remained associated with these post-EEA1 endosomes. Eighty of $133 \mathrm{E}+\mathrm{NgCAM}+$ endosomes (60\%) displayed a decrease in EEA1 over time, whereas 28 of $133(21 \%)$ showed an increase in EEA1 intensity. E+ endosomes devoid of endocytosed NgCAM also showed changes in EEA1 intensity: 15 of $34(44 \%) \mathrm{E}+$ endosomes decreased EEA1 intensity, whereas 9 of $34(26 \%)$ increased intensity. Occasionally, endocytosed L1/

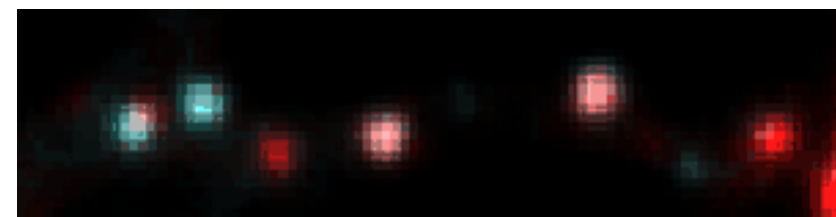

Movie 3. The movie corresponds to Figure $4 \boldsymbol{B}$. GFP-EEA1 and $\mathrm{NgCAM}$ cotransfected into a DIV 9/10 neuron. Alexa Fluor-546-coupled anti-NgCAM antibodies were added to the cells for $30 \mathrm{~min}$ at $37^{\circ} \mathrm{C}$, and live imaging was performed after the antibody was washed out. The acquisition frame rate is 1 frame/s. Playback is 7 frames $/ \mathrm{s}$.

NgCAM was seen to transport in EEA1-negative carriers: only $12 \%$ of all motile compartments contained both EEA1 and $\mathrm{Ng}$ $\mathrm{CAM}$, and among E+NgCAM+ vesicles, $4 \%$ were motile. The exact identity of the EEA1-negative NgCAM carriers is not known, and clear examples of budding events of L1/NgCAMpositive carriers from endosomes could not be observed with our acquisition parameters.

We previously (Yap et al., 2008) performed dual live imaging of NEEP21 and endocytosed NgCAM. NEEP21 mostly colocalized with endocytosed NgCAM in stationary endosomes. Only $18 \%$ of motile NgCAM-positive endosomes also contained NEEP21, and $8 \%$ of motile NEEP21 compartments contained endocytosed NgCAM. Our EEA1/NEEP21 live imaging in this work showed that of all motile NEEP21-positive compartments, $96 \%$ were $\mathrm{E}-/ \mathrm{N}+$. Extrapolating from our two datasets, we conclude that $7-8 \%$ of motile $\mathrm{E}-/ \mathrm{N}+$ carriers contained endocytosed NgCAM. Fewer than $10 \%$ of motile NEEP21-positive vesicles thus contained endocytosed cargo in a $1 \mathrm{~min}$ imaging period. Since we observed stationary $\mathrm{E}-/ \mathrm{N}+$ compartments becoming motile on occasion, some of the stationary $\mathrm{NgCAM}+/ \mathrm{E}-/ \mathrm{N}+$ endosomes might be motile at some point in their life span. The vast majority of $\mathrm{E}-/ \mathrm{N}+$ motile carriers thus might correspond to TGN-derived vesicles containing NEEP21. Alternatively, NEEP21 might recycle in motile $\mathrm{E}-/ \mathrm{N}+$ carriers from endosomes back to the TGN. Future work will address these possibilities.

\section{Discussion}

Endosomal trafficking controls the levels of receptors, regulates many signaling pathways important in development (Yap and Winckler, 2012), and is linked to many human diseases. A better understanding of neuronal endosomes is thus important. We investigated the organization of early endosomes in dendrites of primary hippocampal neurons using live imaging. The early endosomal antigen EEA1 is widely used as a marker for early endosomes, but its dynamic behavior, its relationship with other neuronal endosomal proteins, and its role in regulating axon and dendrite elaboration are not known. Intriguingly, EEA1 is implicated in neurological disease (Selak et al., 2006). Neurons also express a number of neuron-specific endosomal proteins, including NEEP21. NEEP21 has been implicated in regulating AMPA receptor recycling (Steiner et al., 2005), and thus synaptic function, amyloidogenic processing of $\beta$ APP (Norstrom et al., 2010), and trafficking of L1/NgCAM (Yap et al., 2008), but how NEEP2 1 relates to EEA1-containing endosomes in dendrites, and what roles these dendritic endosomes play in trafficking and neuronal growth are not well understood.

How do NEEP21-positive compartments relate to EEA1positive early endosomes dynamically?

Neurons contained both EEA1-positive as well as EEA1-negative NEEP21 endosomes. Dual live imaging of EEA1 and NEEP21 in 
dendrites of primary neurons showed that EEA1 endosomes rarely moved and were long lived on the order of tens of minutes. NEEP21 was also frequently associated with stationary endosomes, but could also be found to move bidirectionally along dendrites. The majority of moving NEEP21 compartments did not contain EEA1. We thus asked how the motile NEEP21 compartments related to stationary EEA1- and NEEP21-positive compartments. Events consistent with fusion events could be observed, suggesting that NEEP21 could be delivered to stationary endosomes by vesicular fusion (Fig. 4C). Whether the NEEP21-positive motile compartments are generated by TGN budding or are derived from another membrane compartment is a question for the future, but we found that only $\sim 8 \%$ of them contain endocytosed cargos.

Second, we investigated the behavior of EEAl on stationary endosomes using live imaging and FRAP analysis. FRAP analysis revealed that different EEA1-positive endosomes showed strikingly different dynamic behaviors, which ranged from stable/not exchanging EEA1 to highly dynamic exchange of EEA1. Whether the dynamics of EEA1 recruitment onto endosomes reflects functional subpopulations is a question to be addressed in the future, but we found that the presence of NEEP21 did not predict either more stable or more dynamic EEA1 recruitment. We also observed spontaneous changes in EEA1 intensity on stationary endosomes, either recruitment (brightening) or dissociation (dimming). Dissociation of EEA1 from a NEEP21-positive endosome was the most frequently observed event ( $62 \%$ of events), indicating that EEA1-positive NEEP21 endosomes $(\mathrm{E}+\mathrm{N}+)$ could convert into NEEP21-positive endosomes (E-/N+; Fig. $4 C)$. This observation is consistent with the reported localization by immuno-EM of NEEP21 in mutivesicular bodies (SabéranDjoneidi et al., 1998; Utvik et al., 2009). In 38\% of events, EEA1 was recruited onto NEEP21-positive compartments (Fig. 4C). Our data thus suggest a model for the early endocytic pathway in dendrites in which EEA1-positive, NEEP21-positive $(\mathrm{E}+\mathrm{N}+)$ endosomes are generated by the following two pathways: EEA1 is recruited onto $\mathrm{E}-/ \mathrm{N}+$ endosomes and/or motile $\mathrm{E}-/ \mathrm{N}+$ vesicles fuse with stationary EEA1+ endosomes. Subsequently, $\mathrm{E}+\mathrm{N}+$ endosomes are consumed by dissociation of EEA1 from $\mathrm{E}+\mathrm{N}+$ endosomes, resulting in post-EE NEEP21-positive endosomes. The subsequent fate and function of these post-EE NEEP21 endosomes remains to be investigated, but our data indicate that endocytosed cargos (e.g., L1/NgCAM) frequently remain associated with the post-EE stationary endosome.

\section{Are axon or dendrite growth dependent on dendritic early endosomes?}

Both EEA1 and NEEP21 are found only in somata and dendrites of primary neurons and are absent from axonal endosomes. We previously found that downregulation of NEEP21 led to missorting of L1/NgCAM to the somatodendritic surface (Yap et al., 2008). We now showed that interference with EEA1 function in early endosome maturation similarly disrupted L1/NgCAM axonal trafficking. Furthermore, we established that interference with endosomal function in dendrites (both EEA1 and NEEP21) led to shorter axons and fewer dendrites in primary neurons cultured on L1-Fc. When neurons were cultured on N-cadherin$\mathrm{Fc}$, we did not observe a significant decrease in axon length when NEEP21 was downregulated, suggesting that the dependence of axon growth on NEEP21 function varies with the substrates and adhesion receptors involved. Since trafficking of L1/NgCAM is disrupted by interference with NEEP21 and EEA1, we propose that the observed outgrowth defects on L1 substrate are the result of aberrant L1/NgCAM trafficking through endosomes.

\section{Conclusions}

Our data demonstrated that interference with EEA1 and NEEP21 function impaired axon outgrowth on L1 substrate, demonstrating the central role of early endosomes and their maturation in the proper development of neurons. Extensive live imaging revealed that EEA1-containing early endosomes in dendrites were mostly stationary, long-lived compartments. NEEP21 was associated with a subset of EEA1-positive early endosomes $(\mathrm{E}+\mathrm{N}+)$, and these double-positive compartments were converted to EEA1-negative, NEEP21-positive endosomes $(\mathrm{E}-/ \mathrm{N}+)$ via EEA1 dissociation. Importantly, the inhibition of endosome maturation with DN-EEA1 decreased the generation of the $\mathrm{E}-/ \mathrm{N}+$ endosome. In addition, events consistent with the fusion of motile NEEP21-positive endosomes with stationary endosomes could be observed, suggesting that NEEP21 could be delivered in vesicles into the early endosome. Live imaging thus made it possible to construct a map of the dynamics of endosomal compartments in dendrites of primary neurons.

\section{References}

Alberi S, Boda B, Steiner P, Nikonenko I, Hirling H, Muller D (2005) The endosomal protein NEEP21 regulates AMPA receptor-mediated synaptic transmission and plasticity in the hippocampus. Mol Cell Neurosci 29: 313-319. CrossRef Medline

Davidson HT, Xiao J, Dai R, Bergson C (2009) Calcyon is necessary for activity-dependent AMPA receptor internalization and LTD in CA1 neurons of hippocampus. Eur J Neurosci 29:42-54. CrossRef Medline

Ha J, Lo KW, Myers KR, Carr TM, Humsi MK, Rasoul BA, Segal RA, Pfister KK (2008) A neuron-specific cytoplasmic dynein isoform preferentially transports TrkB signaling endosomes. J Cell Biol 181:1027-1039. CrossRef Medline

Hoogenraad CC, Popa I, Futai K, Martinez-Sanchez E, Wulf PS, van Vlijmen T, Dortland BR, Oorschot V, Govers R, Monti M, Heck AJ, Sheng M, Klumperman J, Rehmann H, Jaarsma D, Kapitein LC, van der Sluijs P (2010) Neuron specific Rab4 effector GRASP-1 coordinates membrane specialization and maturation of recycling endosomes. PLoS Biol 8:e1000283. CrossRef Medline

Lasiecka ZM, Winckler B (2011) Mechanisms of polarized membrane trafficking in neurons-focusing in on endosomes. Mol Cell Neurosci 48: 278-287. CrossRef Medline

Lasiecka ZM, Yap CC, Caplan S, Winckler B (2010) Neuronal early endosomes require EHD1 for L1/NgCAM trafficking. J Neurosci 30:1648516497. CrossRef Medline

Lawe DC, Patki V, Heller-Harrison R, Lambright D, Corvera S (2000) The FYVE domain of early endosome antigen 1 is required for both phosphatidylinositol 3-phosphate and Rab5 binding. Critical role of this dual interaction for endosomal localization. J Biol Chem 275:3699-3705. CrossRef Medline

Lawe DC, Chawla A, Merithew E, Dumas J, Carrington W, Fogarty K, Lifshitz L, Tuft R, Lambright D, Corvera S (2002) Sequential roles for phosphatidylinositol 3-phosphate and Rab5 in tethering and fusion of early endosomes via their interaction with EEA1. J Biol Chem 277:8611-8617. CrossRef Medline

Muthusamy N, Ahmed SA, Rana BK, Navarre S, Kozlowski DJ, Liberles DA, Bergson C (2009) Phylogenetic analysis of the NEEP21/calcyon/P19 family of endocytic proteins: evidence for functional evolution in the vertebrate CNS. J Mol Evol 69:319-332. CrossRef Medline

Norstrom EM, Zhang C, Tanzi R, Sisodia SS (2010) Identification of NEEP21 as a amyloid precursor protein-interacting protein in vivo that modulates amyloidogenic processing in vitro. J Neurosci 30:1567715685. CrossRef Medline

Ouyang Q, Lizarraga SB, Schmidt M, Yang U, Gong J, Ellisor D, Kauer JA, Morrow EM (2013) Christianson syndrome protein NHE6 modulates TrkB endosomal signaling required for neuronal circuit development. Neuron 80:97-112. CrossRef Medline

Pottier C, Hannequin D, Coutant S, Rovelet-Lecrux A, Wallon D, Rousseau S, 
Legallic S, Paquet C, Bombois S, Pariente J, Thomas-Anterion C, Michon A, Croisile B, Etcharry-Bouyx F, Berr C, Dartigues JF, Amouyel P, Dauchel H, Boutoleau-Bretonnière C, Thauvin C, et al. (2012) High frequency of potentially pathogenic SORL1 mutations in autosomal dominant early-onset Alzheimer disease. Mol Psychiatry 17:875-879. CrossRef Medline

Sabéran-Djoneidi D, Marey-Semper I, Picart R, Studler JM, Tougard C, Glowinski J, Lévi-Strauss M (1995) A 19-kDa protein belonging to a new family is expressed in the Golgi apparatus of neural cells. J Biol Chem 270:1888-1893. CrossRef Medline

Sabéran-Djoneidi D, Picart R, Escalier D, Gelman M, Barret A, Tougard C, Glowinski J, Lévi-Strauss M (1998) A 21-kDa polypeptide belonging to a new family of proteins is expressed in the Golgi apparatus of neural and germ cells. J Biol Chem 273:3909-3914. CrossRef Medline

Selak S, Paternain AV, Fritzler MJ, Lerma J (2006) Human autoantibodies against early endosome antigen-1 enhance excitatory synaptic transmission. Neuroscience 143:953-964. CrossRef Medline

Steiner P, Sarria JC, Glauser L, Magnin S, Catsicas S, Hirling H (2002) Modulation of receptor cycling by neuron-enriched endosomal protein of 21 kD. J Cell Biol 157:1197-1209. CrossRef Medline

Steiner P, Alberi S, Kulangara K, Yersin A, Sarria JC, Regulier E, Kasas S, Dietler G, Muller D, Catsicas S, Hirling H (2005) Interactions between NEEP21, GRIP1 and GluR2 regulate sorting and recycling of the glutamate receptor subunit GluR2. EMBO J 24:2873-2884. CrossRef Medline

Utvik JK, Haglerød C, Mylonakou MN, Holen T, Kropf M, Hirling H, Skare O, Laake P, Ottersen OP, Haug FM, Davanger S (2009) Neuronal en- riched endosomal protein of $21 \mathrm{kDa}$ colocalizes with glutamate receptor subunit GLUR2/3 at the postsynaptic membrane. Neuroscience 158:96104. CrossRef Medline

Wegner CS, Wegener CS, Malerød L, Pedersen NM, Progida C, Bakke O, Stenmark H, Brech A (2010) Ultrastructural characterization of giant endosomes induced by GTPase-deficient Rab5. Histochem Cell Biol 133: 41-55. CrossRef Medline

Wijsman EM, Pankratz ND, Choi Y, Rothstein JH, Faber KM, Cheng R, Lee JH, Bird TD, Bennett DA, Diaz-Arrastia R, Goate AM, Farlow M, Ghetti B, Sweet RA, Foroud TM, Mayeux R (2011) Genome-wide association of familial late-onset Alzheimer's disease replicates BIN1 and CLU and nominates CUGBP2 in interaction with APOE. PLoS Genet 7:e1001308. CrossRef Medline

Wisco D, Anderson ED, Chang MC, Norden C, Boiko T, Fölsch H, Winckler B (2003) Uncovering multiple axonal targeting pathways in hippocampal neurons. J Cell Biol 162:1317-1328. CrossRef Medline

Yap CC, Winckler B (2012) Harnessing the power of the endosome to regulate neural development. Neuron 74:440-451. CrossRef Medline

Yap CC, Wisco D, Kujala P, Lasiecka ZM, Cannon JT, Chang MC, Hirling H, Klumperman J, Winckler B (2008) The somatodendritic endosomal regulator NEEP21 facilitates axonal targeting of L1/NgCAM. J Cell Biol 180:827-842. CrossRef Medline

Yap CC, Lasiecka ZM, Caplan S, Winckler B (2010) Alterations of EHD1/ EHD4 protein levels interfere with L1/NgCAM endocytosis in neurons and disrupt axonal targeting. J Neurosci 30:6646-6657. CrossRef Medline 